\title{
Continuous Path Planning for a Data Harvesting Mobile Server
}

\author{
Jerome Le Ny, Munther A. Dahleh, Eric Feron, Emilio Frazzoli
}

\begin{abstract}
We consider a queueing system composed of queues distributed at fixed locations in a continuous environment and a mobile server serving the jobs in the queues with spatially varying rates. For a fluid model of this system, we provide a necessary and sufficient stabilizability condition. Then we briefly investigate the question of server trajectory optimization for the problem of draining the initial fluid in two queues when no further arrivals occur.
\end{abstract}

\section{INTRODUCTION}

Unmanned Aerial Vehicles (UAVs), are actively used in various civil and military data harvesting missions. Another application under consideration is to use UAVs as communication relays, for example between spatially separated groups on a battlefield [1]. Researchers are now developing numerous models targeted towards these applications for mobile sensor systems, see e.g. [2], [3], [4]. Usually the path planning problem is simplified by considering discrete models where data is transfered from locations in the environment to the mobile elements only when the locations are physically visited by these vehicles. The path of the vehicles is then determined by solving a combinatorial optimization problem, such as the Mobile Element Scheduling Problem [5] or other variations of vehicle routing problems. If this problem is approached from a queueing theory perspective, there is an extensive literature under the rubric of polling systems [6], which is mostly concerned with performance evaluation of open-loop path-planning policies (the queues are served in some fixed cyclic order). The work on polling systems also assumes a discrete model where the service at the queues can be performed only if the server is present at the queue location.

In contrast we consider a continuous polling model where a server can serve spatially separated queues with service rates that depend on the position of the server with respect to the queues. This could model for example the uplink of a wireless transmission system where base stations on the ground transmit their data to a mobile fusion center. Another application concerns planning the trajectory of a mobile measurement station: the quality of the measurements and the time to acquire a bit of information typically depend on the distance to the target. A related integrated model involving path planning and information collection was presented recently in [7]. Our goal is to design the trajectory of the server in order to:

J. Le Ny, M. Dahleh and E. Frazzoli are with the Laboratory for Information and Decision Systems, Massachusetts Institute of Technology, Cambridge, MA 02139-4307, USA jlenyealum.mit.edu, dahleh@mit.edu, frazzoli@mit.edu.

E. Feron is with the School of Aerospace Engineering, Georgia Tech, Atlanta, GA 30332, USA eric.ferondaerospace.gatech.edu

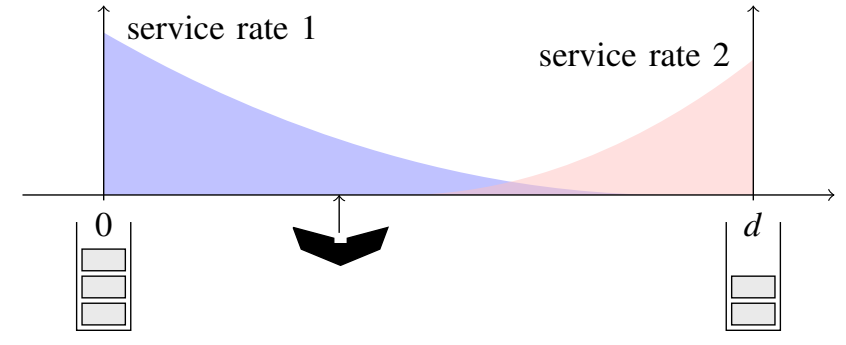

Fig. 1. Uplink from two fixed stations to a mobile receiver, with spatially decaying service rates.

- stabilize the system, i.e., keep the number of jobs in the different queues uniformly bounded.

- optimize a given performance measure, such as minimizing the total number of jobs at all queues over a given horizon.

Discretizing space and treating the problem as a Markov decision process (MDP) leads to intractable computational problems and offers little insight into the design of the server trajectories. Instead, we rely on a deterministic fluid approximation of the problem. Fluid models have proved to be very useful in obtaining insight and policies with good performance in the control of queueing networks [8], including discrete polling systems [9], where more traditional approaches based on MDPs are often too detailed and suffer the curse of dimensionality.

The rest of the paper is organized as follows. Section II describes the fluid model considered. A necessary and sufficient condition for its stabilization, the main result of this paper, is presented in section III. Finally in section IV, we look at the trajectory optimization problem for an example of a draining problem in which the goal is to collect an initial set of packets from the queues as efficiently as possible, without taking into account new arrivals. This model could be useful to develop batch policies ensuring a degree of fairness in service by periodically freezing the jobs to be served next in the queues. A simple performance bound and numerical simulations briefly discuss the relationship between the fluid model and stochastic models assuming variability.

\section{FLUID MODEL}

Fig. 1 presents a conceptual model of the problem for two base stations. Some information to be transmitted to a mobile server arrives at $N$ queues located at fixed sites, with constant rates $\alpha=\left[\alpha_{1}, \ldots, \alpha_{N}\right]^{T}$. We represent this information as a continuous quantity, called fluid hereafter. The fluid present in the queues can be drained by a server which can perform $l$ different on-board activities, controlled by an allocation 
rate vector $\zeta \in \mathbb{R}_{+}^{l}$, subject to certain constraints, potentially dependent on the position $x \in \mathbb{R}^{d}$ of the server. We assume these constraints to be linear in $\zeta$, i.e., for a given position $x$ of the server, $\zeta$ belongs to the convex polyhedron

$$
\zeta \in \mathrm{U}(x)=\{\zeta: \zeta \geq 0, C(x) \zeta \leq 1\},
$$

where $C(x)$ is called the constituency matrix when the server is at position $x$. The server is mobile and can have dynamics. As an example, we assume in section IV that the server has the trivial dynamics with bounded velocity

$$
\dot{x}=u,|u| \leq V, x(0)=x_{0},
$$

where $u$ is the velocity control. Models of the vehicle dynamics much more general than (2) could be used. In fact, the dynamics of the vehicle do not play a role to obtain our stability condition for the fluid model, except for the following assumption. We let $\mathrm{X} \subset \mathbb{R}^{d}$ denote the set of reachable values for the server position $x$. Then we assume throughout this paper that

Assumption 1: The vehicle can stop at an arbitrary location of its reachable space $X$.

The stability condition presented below would have to be adapted to work for vehicles which are not able to stop, such as fixed-wing UAVs, unless the size of the loitering pattern can be neglected. Note moreover that although the vehicle's dynamics do not play a role in the system's stability analysis, they influence the delay in servicing the jobs in the queues and hence the performance of the system.

The vector of queue sizes $q \in \mathbb{R}_{+}^{N}$ obeys the system of differential equations

$$
\frac{d^{+}}{d t} q(t)=\alpha+B(x(t)) \zeta(t)
$$

where for all $x, B(x)$ is an $N$-by- $l$ matrix. In addition, the state $q$ of the queues is constrained to belong to a polyhedral state-space $Q$, which includes the non-negativity constraints and potential finite buffer constraints. In addition to (2), there are additional implicit constraints on $\zeta$ when $q$ hits the boundaries of $Q$, to prevent the queue lengths from violating their positivity constraints.

Example 2: Consider the two queue example of Fig. 1, and suppose that the two base stations transmit a signal to the mobile server (UAV) over a shared additive white Gaussian noise channel, with power $P_{i}, i=1,2$. The server, when at position $x$, receives the signal from station $i$ with power $p_{i}(x)=P_{i} /\left(C_{i}+\left\|x-x_{i}\right\|\right)^{\beta_{i}}$, where $C_{i}$ and $\beta_{i}$ are constants, and $x_{i}$ is the position of the $i^{\text {th }}$ base station. If the server decodes the transmissions of the base stations sequentially, it can achieve communication rates $R_{i}(x)$ that are subject to the following constraints [10]:

$R_{i} \leq \log \left(1+p_{i}(x)\right), i=1,2 ; R_{1}+R_{2} \leq \log \left(1+p_{1}(x)+p_{2}(x)\right)$.

To put this example in our framework, we can let $\mu_{i}(x)=$ $\log \left(1+p_{i}(x)\right)$ for $i=1,2, R_{i}=\mu_{i}(x) \zeta_{i}$ and rewrite the constraints

$$
0 \leq \zeta_{i} \leq 1, i=1,2, \quad \text { and } \frac{\mu_{1}(x) \zeta_{1}+\mu_{2}(x) \zeta_{2}}{\log \left(1+p_{1}(x)+p_{2}(x)\right)} \leq 1,
$$

which are of the form (1). The matrix $B$ in this case is a 2-by-2 diagonal matrix with $B_{i i}(x)=-\mu_{i}(x), i=1,2$.

\section{Stability of The Fluid Model}

In general, it is not possible to drain all queues to zero simultaneously using the mobile server, if the intersection of the draining regions, which are the regions of $X$ where the server can drive the queue level of a particular queue towards 0 , is empty. Hence we use the following weaker notion of stability.

Definition 3: The system $q$ is said to be stabilizable if there exist constants $K$ and $T$ such that, for any initial condition $q_{0}$,

$$
\|q(t)\|_{\infty} \leq K, \forall t \geq T\left\|q_{0}\right\|_{\infty} .
$$

To study the possible trajectories of the vector $q$ in (3), we will study the velocity space for the queues, generalizing the corresponding definitions in [8]. Let

$$
\mathrm{Y}=\bigcup_{x \in \mathrm{X}}\{\{x\} \times \mathrm{U}(x)\} .
$$

First we note that a trajectory $(x(t), \zeta(t))$ defines for each $T \in \mathbb{R}_{+}$a probability measure $P_{T}^{x, \zeta}$ on $\mathrm{Y}$ where $P_{T}^{x, \zeta}(A)$ is the proportion of time that the pair $(x, \zeta)$ spends in set $A \subset \mathrm{Y}$ in the interval $[0, T]$. In other words

$$
P_{T}^{x, \zeta}(A)=\frac{1}{T} \int_{0}^{T} \delta_{x(t), \zeta(t)}(A) d t
$$

Then, integrating (3), we obtain, for all $T \in \mathbb{R}_{+}$,

$$
\begin{aligned}
& q(T)=q_{0}+\alpha T+\int_{0}^{T} B(x(t)) \zeta(t) d t \\
& q(T)=q_{0}+\alpha T+\int_{0}^{T} \int_{Y} B(x) \zeta \delta_{x(t), \zeta(t)}(d x, d \zeta) d t \\
& q(T)=q_{0}+\alpha T+T \int_{Y} B(x) \zeta P_{T}^{x, \zeta}(d x, d \zeta) .
\end{aligned}
$$

Define the velocity space for the queues as

$\mathrm{V}=\left\{\alpha+\int_{\mathrm{Y}} B(x) \zeta P(d x, d \zeta): P\right.$ a probability measure on $\left.\mathrm{Y}\right\}$

Clearly $\mathrm{V}$ is a convex set in $\mathbb{R}^{N}$. It is the set of directions in which we can steer the vector $q$, by moving the server and controlling the allocation rates $\zeta$, if we do not take into account the constraints imposed on the vehicle dynamics. In fact, we will see below that these constraints do not play a role as far as stability is concerned.

Now the following is useful for approximating the set $\mathrm{V}$. Note that by definition of the Lebesgue integral, we can approximate the vector $\alpha+\int_{Y} B(x) \zeta d P(x, \zeta)$ arbitrarily closely by the "Lebesgue sums"

$$
\alpha+\sum_{k=1}^{n} B\left(x^{k}\right) \zeta^{k} p^{k}
$$

where $\sum_{k=1}^{n} p^{k}=1$ (since $\left.P(\mathrm{Y})=1\right), x^{k} \in \mathrm{X}$, and $\zeta^{k} \in$ $\mathrm{U}\left(x^{k}\right), i=1, \ldots, n$. Such a sum correspond to a point in $\mathrm{V}$ with associated measure $\sum_{k=1}^{n} p^{k} \delta_{x^{k}, \zeta^{k}}$. Let

$$
\hat{\mathrm{V}}(x)=\{\alpha+B(x) \zeta: \zeta \in \mathrm{U}(x)\}
$$


For each position $x$ of the server, $\hat{\mathrm{V}}(x)$ is a convex polyhedron. Note that all these polyhedrons contain the point $\alpha$, since $0 \in \mathrm{U}(x)$ for all $x$. Then we define

$$
\hat{V}=\operatorname{conv}\left\{\bigcup_{x \in X} \hat{V}(x)\right\} .
$$

Clearly $\hat{V}$ is the set of points of the form (5), and so $\hat{V}$ is dense in $V$. Since $\hat{V}$ and $V$ are convex, this implies

$$
\operatorname{int}(\hat{\mathrm{V}})=\operatorname{int}(\mathrm{V}) \text {. }
$$

Let $C^{\varepsilon}$ be the $l^{\infty}$ ball

$$
C^{\varepsilon}=\left\{v \in \mathbb{R}^{N}:-\varepsilon<v_{i} \leq 0, i=1, \ldots, N\right\} .
$$

Then the following theorem provides a necessary and sufficient stabilizability condition for the fluid model.

Theorem 4: The following conditions are equivalent

1) The fluid model (3) is stabilizable.

2) $C^{\varepsilon} \subset \mathrm{V}$, for some $\varepsilon$.

3) $C^{\varepsilon} \subset \hat{V}$, for some $\varepsilon$.

The proof of theorem 4 can be found in the appendix. The most useful part of the theorem is the equivalence between conditions 1 and 3 . We present here a simple lowdimensional example to clarify the geometric picture.

Example 5: Consider a scheduling problem where the server can move on the real line and serve two queues placed at position 0 and $d$ with service rates $\mu_{1}(x) \zeta_{1}$ and $\mu_{2}(x) \zeta_{2}$ respectively, subject only to the constraints $0 \leq \zeta_{1}, \zeta_{2} \leq 1$. Clearly in this case we should always let $\zeta_{i}=1, i=1,2$. The arrival rates are $\alpha_{1}$ and $\alpha_{2}$ respectively. The queue dynamics are then

$$
\begin{aligned}
& {\left[\begin{array}{l}
\dot{q}_{1} \\
\dot{q}_{2}
\end{array}\right]=\left[\begin{array}{l}
\alpha_{1} \\
\alpha_{2}
\end{array}\right]+\left[\begin{array}{cc}
-\mu_{1}(x) & 0 \\
0 & -\mu_{2}(x)
\end{array}\right]\left[\begin{array}{l}
\zeta_{1} \\
\zeta_{2}
\end{array}\right],} \\
& \mathrm{U}(x)=\mathrm{U}=\left\{\zeta=\left[\zeta_{1} \zeta_{2}\right]^{T}: 0 \leq \zeta_{1}, \zeta_{2} \leq 1\right\} \forall x,
\end{aligned}
$$

and in addition, we have the positivity constraints $q_{1}, q_{2} \geq 0$. Fig. 2 shows the velocity set $\hat{V}$ for an instance of the problem where

$$
\alpha_{1}=1, \alpha_{2}=3, \mu_{1}(x)=\frac{3}{1+|x|}, \mu_{2}(x)=\frac{4}{1+|x-4|} .
$$

Let us define the draining region of queue $i$ as

$$
D^{i}:=\left\{x \in \mathbb{R} \mid \alpha_{i}-\mu_{i}(x)<0\right\}, \quad i \in\{1,2\},
$$

and assume that $D^{1} \cap D^{2}=\emptyset$. This corresponds to the situation of fig. 2 , since the curve $\left\{\left(\alpha_{1}-\mu_{1}(x), \alpha_{2}-\mu_{2}(x)\right)\right.$ : $x \in \mathbb{R}\}$ does not intersect the set $\operatorname{int}\left(\mathbb{R}_{-}^{2}\right)$. The geometric condition for stability of theorem 4 can then be translated into an algebraic condition. Indeed we see from the theorem and the figure that the system is stable if and only if there exist two points $x^{1} \in D^{1}$ and $x^{2} \in D^{2}$ and a parameter $0<\theta<1$ such that

$$
\theta\left(\begin{array}{c}
\alpha_{1}-\mu_{1}\left(x^{1}\right) \\
\alpha_{2}-\mu_{2}\left(x^{1}\right)
\end{array}\right)+(1-\theta)\left(\begin{array}{c}
\alpha_{1}-\mu_{1}\left(x^{2}\right) \\
\alpha_{2}-\mu_{2}\left(x^{2}\right)
\end{array}\right)<\left(\begin{array}{c}
0 \\
0
\end{array}\right) .
$$

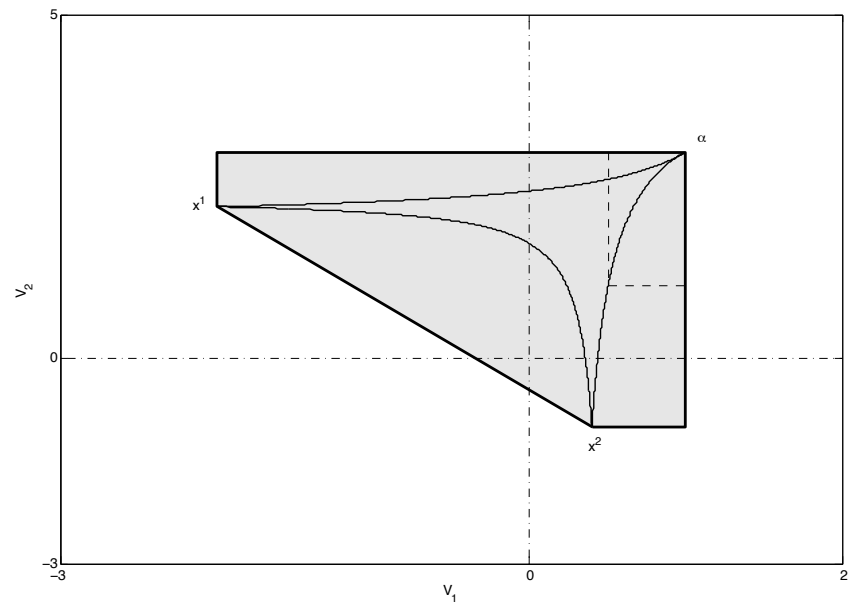

Fig. 2. Velocity set $\hat{V}$ (filled polygon) for the two-dimensional example 5 . The curve is the set of points $\left\{\left(\alpha_{1}-\mu_{1}(x), \alpha_{2}-\mu_{2}(x)\right): x \in \mathbb{R}\right\}$. We also show a rectangle which is the set $\mathrm{V}(x)$ for some value of $x$. From the figure and theorem 4 , we see immediately that the system is stabilizable since $(0,0)$ is an interior point of the velocity set. Moreover, we can approximately steer the vector $q$ in the directions contained in $\mathbb{R}_{-}^{2}$ using policies that switch between the points $x^{1}$ and $x^{2}$ only. The proportion of time spent at each point determines a convex combination of the velocity vectors at $x^{1}$ and $x^{2}$.

We rewrite these conditions as

$$
\begin{gathered}
\left\{\begin{array}{l}
\alpha_{1}-\mu_{1}\left(x^{2}\right)<\theta\left(\mu_{1}\left(x^{1}\right)-\mu_{1}\left(x^{2}\right)\right) \\
\alpha_{2}-\mu_{2}\left(x^{1}\right)<(1-\theta)\left(\mu_{2}\left(x^{2}\right)-\mu_{2}\left(x^{1}\right)\right)
\end{array}\right. \\
\text { to obtain: } \exists \theta \in(0,1) \text { s.t. }\left\{\begin{array}{l}
\frac{\alpha_{1}-\mu_{1}\left(x^{2}\right)}{\mu_{1}\left(x^{1}\right)-\mu_{1}\left(x^{2}\right)}<\theta \\
\frac{\alpha_{2}-\mu_{2}\left(x^{1}\right)}{\mu_{2}\left(x^{2}\right)-\mu_{2}\left(x^{1}\right)}<1-\theta .
\end{array}\right.
\end{gathered}
$$

These last condition is equivalent to the following condition: there exists $\left(x^{1}, x^{2}\right) \in D^{1} \times D^{2}$ such that

$$
\frac{\alpha_{1}-\mu_{1}\left(x^{2}\right)}{\mu_{1}\left(x^{1}\right)-\mu_{1}\left(x^{2}\right)}+\frac{\alpha_{2}-\mu_{2}\left(x^{1}\right)}{\mu_{2}\left(x^{2}\right)-\mu_{2}\left(x^{1}\right)}<1,
$$

which, after expansion and simplifications, can also be rewritten:

$$
\exists x^{1} \in D^{1}, x^{2} \in D^{2}, \text { s.t. } \frac{\left(\alpha_{1}-\mu_{1}\left(x^{2}\right)\right)\left(\alpha_{2}-\mu_{2}\left(x^{1}\right)\right)}{\left(\mu_{1}\left(x^{1}\right)-\alpha_{1}\right)\left(\mu_{2}\left(x^{2}\right)-\alpha_{2}\right)}<1 .
$$

Example 6: Let $\quad \alpha_{1}=0.5, \alpha_{2}=0.55, \mu_{1}(x)=$ $\frac{1}{1+0.1 x^{2}}, \mu_{2}(x)=\frac{1}{1+0.1|x-8|^{3}}$. One can then verify that $D_{1} \cap D_{2}=\emptyset$. Condition (8) for $x^{1}=0$ and $x^{2}=8$ gives $0.86<1$ hence the system is stable. Looking only at the condition $\alpha_{1} / \mu_{1}(0)+\alpha_{2} / \mu_{2}(8)=1.05$, we see that the system is easier to stabilize than the traditional discrete two-queue setup, for which $\mu_{1}\left(x^{2}\right)=\mu_{2}\left(x^{1}\right)=0$.

\section{TRAJectory Optimization For a Draining PROBLEM}

We consider now a server trajectory optimization problem for the configuration of example 5 , with the queue dynamics given by (7). In this case we can set $\zeta_{1}=\zeta_{2}=1$. We study the problem of draining optimally the fluid present initially in the system, when no new arrival occurs (i.e., $\alpha_{1}=\alpha_{2}=0$ ) and we incur a running linear cost $c(q(t)):=c_{1} q_{1}(t)+c_{2} q_{2}(t)$, with 
$c_{1}, c_{2}$ positive constants. We wish to minimize the infinitehorizon cost

$$
J\left(q_{0} ; u(\cdot)\right)=\int_{0}^{\infty} c_{1} q_{1}(t)+c_{2} q_{2}(t) d t,
$$

for a server with the simple dynamics (2) and bounded velocity. The case of infinite velocity and its solution using a modification of the well known " $c \mu$ rule" is presented in [11].

\section{A. Necessary Conditions for Optimality}

Using the minimum principle, we can derive necessary conditions satisfied by optimal trajectories. For each subset $S$ of $\{1,2\}$, define the Hamiltonian

$$
H^{S}(q, x, u, p)=\sum_{i \in S} c_{i} q_{i}-\sum_{i \in S} p_{i} \mu_{i}(x)+p_{0} u,
$$

which is the Hamiltonian for the system where $S$ represents the set of non-empty queues, and $p=\left[p_{0}, p_{1}, p_{2}\right]$ are the adjoint variables. In contrast to standard optimal control problems for fluid models, where the draining rate is assumed to be under direct control, we cannot enforce the positivity constraint on the fluid levels by a simple state dependent constraint on the control. We must take into account the discontinuity in the dynamics of the system when a queue level reaches zero.

Suppose that both queues are non-empty, that the server is in the draining region $D_{i}$ of queue $i$, and that queue $i$ becomes empty at some time $\tau_{i}$. Suppose that there is only one queue becoming empty at time $\tau_{i}$. From the results in [12, sections 3.5 and 3.6], at the boundary $q_{i}=0$, we have the following relations

$$
\begin{array}{rr}
p_{i}\left(\tau_{i}^{-}\right)=p_{i}\left(\tau_{i}^{+}\right)+\pi_{i}, \quad p_{j}\left(\tau_{i}^{-}\right)=p_{j}\left(\tau_{i}^{+}\right), & j \neq i \\
H^{\{1,2\}}\left(\tau_{i}^{-}\right)=H^{\{j\}}\left(\tau_{i}^{+}\right), & j \neq i
\end{array}
$$

where $\pi_{i}$ is a (constant) Lagrange multiplier. Now using the continuity of the state variables $q, x$ and of the Hamiltonian (11), we get $p_{i}\left(\tau_{i}^{-}\right) \mu_{i}\left(x\left(\tau_{i}^{-}\right)\right)=0$, and since the server must be in the draining region $D_{i}$ (where $\mu_{i}\left(x\left(\tau_{i}^{-}\right)\right)>0$ ) when queue $i$ becomes empty, this implies

$$
p_{i}\left(\tau_{i}^{-}\right)=0 .
$$

Hence, the adjoint variable corresponding to queue $i$ vanishes at the time when the queue becomes empty. The optimal control is of the bang-bang type

$$
u(t)=-\operatorname{sign}\left(p_{0}(t)\right) V
$$

When the queues in the set $S$ are non-empty, the adjoint equations are,

$$
\dot{p}_{i}=-c_{i}, \quad i \in S ; \quad \dot{p}_{0}=\sum_{i \in S} p_{i} \frac{d \mu_{i}}{d x}(x(t)),
$$

where the derivative with respect to $x$ can be taken to mean a subgradient in the case of nonsmooth rate functions. From the first set of equations and the preceding argument, we deduce

$$
p_{i}(t)=c_{i}\left(\tau_{i}-t\right), \quad i \in\{1,2\}
$$

\begin{tabular}{|c|c|c|}
\hline$R<1$ & $\tau_{1, r} \leq \tau_{2, r}$ & $u=+V$ \\
& $\tau_{1, r}>\tau_{2, r} \wedge \tau_{2, l}>R \tau_{1, l}$ & $u=+V$ \\
& $\tau_{1, r}>\tau_{2, r} \wedge \tau_{2, l} \leq R \tau_{1, l}$ & $u=-V$ \\
\hline$R>1$ & $\tau_{2, l} \leq \tau_{1, l}$ & $u=-V$ \\
& $\tau_{2, l}>\tau_{1, l} \wedge \tau_{2, r}<R \tau_{1, r}$ & $u=-V$ \\
& $\tau_{2, l}>\tau_{1, l} \wedge \tau_{2, r} \geq R \tau_{1, r}$ & $u=+V$ \\
\hline
\end{tabular}

TABLE I

OPTIMAL FEEDBACK CONTROL LAW.

where $\tau_{i}$ is the time when queue $i$ empties. Finally, since the dynamics and cost function are independent of time and this is a free terminal time problem, we know that the Hamiltonian is constant and equal to zero along optimal trajectories.

\section{B. Draining Problem with Two Distant Sites and Linear Rate Functions}

We can apply the necessary optimality conditions to obtain a feedback control law for the server. We assume that the server starts initially on the line segment between two base stations located at positions 0 and $d$. We also assume for simplicity that the stations are sufficiently far from the initial position of the server, so that the queue at each base station would become empty before the server can reach it, if it were to always move towards that base station. The objective is to drain the queues while minimizing the infinite-horizon cost function (9). We assume that the draining region of each queue covers the whole segment $[0, d]$, so that even an immobile server is draining the queues in finite time. In particular, in the region $[0, d]$ of interest, we have

$$
\mu_{1}(x)=\mu_{10}-s_{1} x, \quad \mu_{2}(x)=\mu_{20}-s_{2}(d-x), \quad x \in[0, d],
$$

where $s_{1}$ and $s_{2}$ are constants.

Calling the terminal time $t_{f}$, we have $t_{f}=\tau_{1}$ or $t_{f}=\tau_{2}$ and also

$$
H\left(t_{f}\right)=-V\left|p_{0}\left(t_{f}\right)\right|=0 \Rightarrow p_{0}\left(t_{f}\right)=0 .
$$

We can then determine the optimal trajectories by considering different cases based on which queue becomes empty first. The detailed calculations are provided in [11], and we present here only the resulting control law for the server. Define $R:=\sqrt{\frac{c_{1} s_{1}}{c_{2} s_{2}}}$. We consider only the case $R \neq 1$. Also, let

$$
\begin{aligned}
\tau_{1, l}(x, q) & =\frac{\left(s_{1} x-\mu_{10}\right)+\sqrt{\left(s_{1} x-\mu_{10}\right)^{2}+2 s_{1} V q_{1}}}{s_{1} V} \\
\tau_{2, l}(x, q) & =\frac{-\left(s_{2}(d-x)-\mu_{20}\right)-\sqrt{\left(s_{2}(d-x)-\mu_{20}\right)^{2}-2 s_{2} V q_{2}}}{s_{2} V} \\
\tau_{1, r}(x, q) & =\frac{\left(\mu_{10}-s_{1} x\right)-\sqrt{\left(s_{1} x-\mu_{10}\right)^{2}-2 s_{1} V q_{1}}}{s_{1} V} \\
\tau_{2, r}(x, q) & =\frac{\left(s_{2}(d-x)-\mu_{20}\right)+\sqrt{\left(s_{2}(d-x)-\mu_{20}\right)^{2}+2 s_{2} V q_{2}}}{s_{2} V} .
\end{aligned}
$$

Then Table I summarizes the optimal feedback control law for the draining problem of this section. 

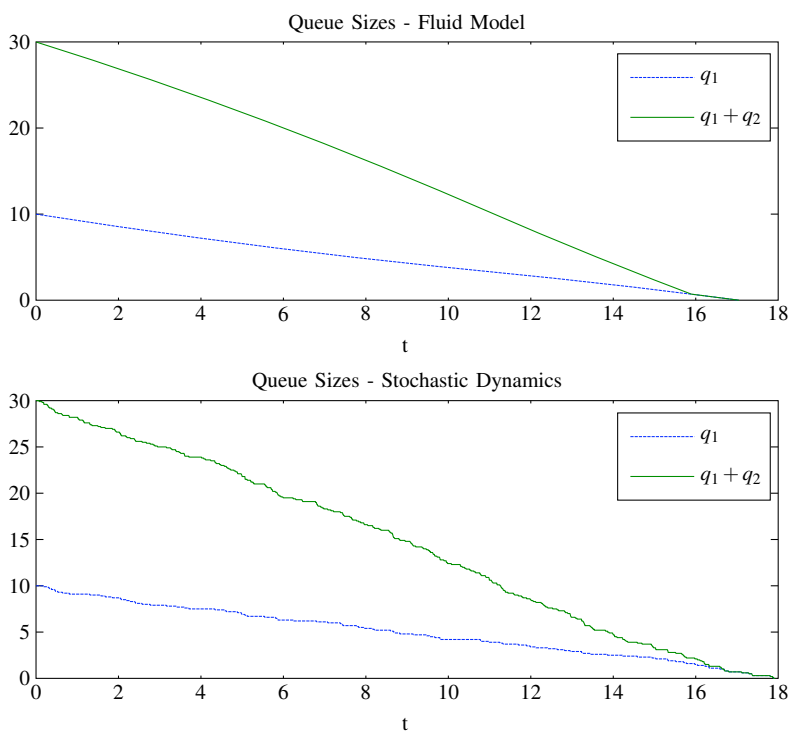

Fig. 3. Examples of trajectories of the queues for the stochastic draining problem with Bernoulli service variables, and for its deterministic fluid approximation (top). The optimal feedback policy of the fluid model was used in both cases to control the server.

\section{Simulation}

Suppose that when we sample the system dynamics with sampling period $T_{s}$, we obtain in fact the following discrete time stochastic evolution

$$
\begin{aligned}
& Q_{i}\left((k+1) T_{s}\right):=Q_{i}(k+1)=Q_{i}(k)+A_{i}(k+1)-B_{i}(k+1 ; X(k)), \\
& X\left((k+1) T_{s}\right):=X(k+1)=X(k)+U(k) T_{s}, \quad|U(k)| \leq V,
\end{aligned}
$$

where the queue lengths $Q_{i}$ are also subject to the nonnegativity constraints. Assume that the arrival process $\{A(k)\}$ is i.i.d. with $E\left[A_{i}(k+1)\right]=\alpha_{i} T_{s}$, and the variables $B_{i}(k+$ $1, x(k))$ are independent, with distribution depending on the position $x(k)$ of the server, and $E\left[B_{i}(k+1 ; X(k))\right]=$ $\mu_{i}(X(k)) T_{s}$. Then, modifying slightly the proof in $[8$, section 4.4], we can prove, if $\mu(x)$ is sufficiently smooth, the following lower bound on the achievable performance for the stochastic system, for each fixed $T \in T_{s} \mathbb{Z}$ :

$$
\inf E\left[\sum_{k=0}^{T / T_{S}} T_{s} c(Q(k))\right] \geq \inf \int_{0}^{T} c(q(t)) d t+o(1),
$$

where the infimum is over all admissible control policies, $Q(0)=q(0)=q_{0}$, and $\lim _{T_{s} \rightarrow 0} o(1)=0$.

Moreover, as the size of the jumps $A(k), B(k)$ becomes small, while keeping the same rates $\alpha_{i}, \mu_{i}(x)$, we expect the stochastic system behavior to approach the behavior of the fluid system (see, e.g., [13]). Fig. 3 gives an example of queue trajectories obtained for a stochastic system of the form (7) and for its fluid approximation, using in both cases the optimal feedback policy computed for the fluid model in the previous section.

\section{CONClusion}

We have presented a trajectory optimization problem for a server serving remotely the jobs arriving at spatially distributed queues. Various research directions and extensions are possible, such as studying the trajectory optimization problem for the average and discounted cost criteria when arrivals occur and for more complicated service rate profiles, geometries, and vehicle dynamics, developing approximate solutions based on dynamic programming techniques, etc. There is a growing literature on the subject of dynamic task assignment for automated vehicle systems [4]. We believe that fluid models such as the one presented here offer a flexible modeling tool to obtain insight into more complex versions of these problems, much as they are proving to be a useful tool for network control.

\section{ACKNOWLEDGMENTS}

The authors thank Sean Meyn for his help generalizing a preliminary version of theorem 4 .

\section{APPENDIX: PROOF OF THEOREM 4}

$1 \Rightarrow 2$. By (4), we have $q_{i}(t)=q_{i}(0)+v_{i}^{t} t, i=1, \ldots, N$ for some $v^{t} \in \mathrm{V}$. Hence $\frac{q_{i}(t)-q_{i}(0)}{t} \in \mathrm{V}$, for all $t>0$. Recall the definition of the constants $K, T$ in definition 3 . Let $\delta \in \mathbb{R}_{+}^{N}$ with $\|\delta\|_{\infty}=1 / T$, and let $q^{n}(t)$ be a stable trajectory with $q^{n}(0)=n \delta, n \in \mathbb{N}$. Then for all $t \geq T n\|\delta\|_{\infty}=n$, we have $\left\|q^{n}(t)\right\|_{\infty} \leq K$. In particular we deduce that for all $n \in \mathbb{N}$,

$$
\frac{q^{n}(n)}{n}-\delta \in V
$$

and so since the sequence $q^{n}(n)$ is bounded by $K$, we see that $-\delta \in \operatorname{cl}(\mathrm{V})$. Moreover if in (13) we take $\delta=$ $\frac{1}{T} e^{i}$, with $e^{i}$ the $i^{\text {th }}$ standard basis vector, we obtain a sequence $\left\{\left(\varepsilon_{1}^{n}, \ldots,-\frac{1}{T}+\varepsilon_{i}^{n}, \ldots, \varepsilon_{N}^{n}\right)\right\}_{n}$ of points of $\mathrm{V}$ with $\varepsilon_{j}^{n} \geq 0, \forall j, n$. Hence by convexity the points $\left(-\frac{1}{T}+\eta\right) e^{i}$ also belong to $\mathrm{V}$, for any $\eta>0$. Finally recalling that $\alpha \in \mathrm{V}$ (with $\alpha \in \mathbb{R}_{+}^{N}$ ) and again by convexity of $\mathrm{V}$, we obtain that $C^{\varepsilon} \subset \vee$ for $\varepsilon=1 / T$.

$2 \Rightarrow 3$. Suppose $C^{\varepsilon} \subset \mathrm{V}$, for some $\varepsilon$. If $C^{\varepsilon} \backslash\{0\} \subset \operatorname{int}(\mathrm{V})$, then $C^{\varepsilon} \subset \hat{\mathrm{V}}$ using (6), $\alpha \in \hat{\mathrm{V}}$ and the convexity of $\hat{\mathrm{V}}$. The rest of the argument concerns the technical point which arises when facets of $C^{\varepsilon}$ are on the boundary of $\mathrm{V}$. We prove that in this case, these facets are also in $\hat{V}$. So assume that there is a point $v^{0} \in C^{\varepsilon} \backslash\{0\}$ which is on the boundary of $V$. We call $\mathscr{P}$ the set of probability measures on $\mathrm{Y}$. We also define, for $P \in \mathscr{P}$,

$$
v(P)=\alpha+\int_{Y} B(x) \zeta P(d x, d \zeta) .
$$

Then let $P^{0} \in \mathscr{P}$ be such that $v\left(P^{0}\right)=v^{0}$.

Since $V$ is convex, there is a supporting hyperplane $\mathrm{H} \subset \mathbb{R}^{N}$ of $\mathrm{V}$ passing through $v_{0}$. By a transformation of coordinates, we can assume that $\mathrm{H}$ is the plane $x_{N}=0$, and that for all $P \in \mathscr{P}$, we have the coordinate $[v(P)]_{N} \leq 0$. Considering Dirac measures, we have that for all $(x, \zeta) \in \mathrm{Y}$, $(\alpha+B(x) \zeta)_{N} \leq 0$. In particular, taking $\zeta=0$ implies that $\alpha_{N}=0$, and so

$$
\forall(x, \zeta) \in \mathrm{Y},(B(x) \zeta)_{N} \leq 0
$$


Now we have

$$
v_{N}^{0}=\int_{Y}(B(x) \zeta)_{N} P^{0}(d x, d \zeta)=0,
$$

which implies by (14) that, except possibly for a set of $Y$ of $P^{0}$-measure 0 , we have $(B(x) \zeta)_{N}=0$. Hence we can restrict the problem of approximating the integral defining $v^{0}$ by using points $B\left(x^{k}\right) \zeta^{k}$ which are on the hyperplane $H$. Repeating the same argument for an intersection of hyperplanes if there is a lower dimensional facet of $C^{\varepsilon}$ (such as an edge) on the boundary of $\mathrm{V}$, we see that we can always restrict the approximation problem for points $v$ of the facet by using points $B\left(x^{k}\right) \zeta^{k}$ that are on that facet. Hence the facet also belongs to $\hat{V}$ (by convexity of $\hat{V}$ again). So $C^{\varepsilon} \subset \hat{V}$.

$3 \Rightarrow 1$. The idea is intuitively the following. For $T$ large enough, $-\frac{q_{0}}{T}$ belongs to $\hat{\mathrm{V}}$, so we can write

$$
0=q_{0}+\alpha T+T \sum_{k=1}^{n} B\left(x^{k}\right) \zeta^{k} p^{k}
$$

Then a policy which moves the server successively through the points $x^{k}, k=1, \ldots, n$, uses at these points the allocation rates $\zeta^{k}$, and spends time $p^{k} T$ at point $x^{k}$ drives the system approximately to 0 if $T$ is much greater than the time spent traveling between the points $x^{k}$. Moreover, this policy is approximately time optimal, since on average it drives the vector of queues towards 0 along the direction $-q_{0}$. Of course, this reasoning is valid only for the case where $q_{0, i}$ is large for all $i$, for which the positivity constraints and the travel times can indeed be neglected. The argument is also complicated by the fact that the choice of the configurations $\left(x^{k}, \zeta^{k}\right)$, which determines the total travel time and the impact of the positivity constraints, depends on the travel direction $-q_{0} / T$ and it becomes problematic to obtain an upper bound $K$ on the steady-state sizes of the queues which is independent of $q_{0}$.

Hence we will show stability by driving the queues toward 0 using a single direction (the diagonal), sacrificing time optimality in the process. Let $q_{0}=\left(q_{0,1}, \ldots, q_{0, N}\right)$ be the initial queue sizes. We start by bringing the queue size vector close to the diagonal $\mathrm{D}:=\left\{q \in \mathbb{R}^{N}: q_{1}=q_{2}=\ldots=q_{N}\right\}$, as follows. For each $j \in\{1, \ldots, N\}$, there is a configuration $\left(x^{j}, \zeta^{j}\right) \in \mathrm{Y}$ such that $v^{j}=B\left(x^{j}\right) \zeta^{j} \in \hat{\mathrm{V}}$ verifies

$$
v_{j}^{j}<0, \text { and } v_{i}^{j} \geq 0, i=1, \ldots, N .
$$

This is easily seen from the facts that $C^{\varepsilon} \subset \hat{V}$ and $\hat{V}$ is convex. Moreover, we can choose these $N$ vectors in such a way that $C^{\varepsilon}$ is contained in the convex cone that they generate. Then there exist nonnegative constants $t^{j}, j=1, \ldots, N$, such that $q_{0}+\sum_{j=1}^{N} t^{j} v^{j}=: q_{1} \in \mathrm{D}$, and moreover, if the server is spending time $t^{j}$ in configuration $v^{j}, j=1, \ldots, N$, the positivity constraints on $q$ never become active. Now if we call $T_{1}$ the time necessary for switching from $v^{1}$ to $v^{2}$, then to $v^{3}$, etc., until $v^{N}$, we can fix $\zeta=0$ while the server travels, and the policy above in fact brings the vector $q$ to the value $q_{1}+\alpha T_{1}$ on the line $\alpha T_{1}+\mathrm{D}$, using a trajectory that never hits the boundaries of $\mathbb{R}_{+}^{N}$. This first phase can be accomplished in a time upper bounded by $\alpha T_{1}+T_{2}\left\|q_{0}\right\|_{\infty}$, where $T_{2}$ is a constant independent of $\left\|q_{0}\right\|_{\infty}$ (this is because the distance from $q_{0}$ to $\mathrm{D}$ is proportional to $\left\|q_{0}\right\|_{\infty}$, and the coefficients $t^{j}$ are of the same order).

Once the vector $q$ is at $q_{1}+\alpha T_{1}$, i.e., close to the diagonal, we drive it toward 0 . More precisely, there is a constant $T_{3}>0$ such that

$$
-\frac{q_{1}}{T_{3}} \in \hat{\mathrm{V}}, \text { so }-q_{1}=\alpha T_{3}+T_{3} \sum_{k=1}^{n} B\left(x^{k}\right) \zeta^{k} p^{k},
$$

for some configurations $\left(x^{k}, \zeta^{k}\right), k=1, \ldots, n$ that are independent of $q_{1}$. Then by adapting the usual the policy that puts the server in the successive configurations $\left(x^{k}, \zeta^{k}\right), k=$ $1, \ldots, n$, we can move the queue size vectors by $-q_{1}+\delta+$ $\alpha T_{4}$. Here $\delta$ is a nonnegative fixed quantity which takes into account the effect of the positivity constraints, and $T_{4}$ is the sum of the switching times between configurations. So at the end of the second step, the vector $q$ is at the point $\delta+$ $\alpha\left(T_{1}+T_{4}\right)$. The duration of the second phase is proportional to $\left\|q_{1}\right\|_{\infty}$, which can be bounded by a constant multiple of $\left\|q_{0}\right\|_{\infty}$. So the total time can be written $\tilde{T}\left\|q_{0}\right\|_{\infty}+\alpha T_{1}$. Using the ideas above, one can see that there are constants $K$ and $K_{1}$, with $K \geq K_{1} \geq\left\|\delta+\alpha\left(T_{1}+T_{4}\right)\right\|$, such that if $\left\|q\left(t_{0}\right)\right\|_{\infty} \leq K_{1}$ for some $t$, we can insure $\|q(t)\|_{\infty} \leq K, \forall t \geq t_{0}$ (we can use the velocity vectors $v^{j}$ of (15) for this purpose). Hence we are done by choosing $T=\tilde{T}+\frac{\alpha}{K_{1}} T_{1}$ in the case $\left\|q_{0}\right\|_{\infty} \geq K_{1}$.

\section{REFERENCES}

[1] "Unmanned aircraft systems roadmap 2005-2030," Office of the Secretary of Defense, Tech. Rep., 2005. [Online]. Available: http://www.acq.osd.mil/usd/Roadmap $\backslash \% 20$ Final2.pdf

[2] Y. Gu, D. Bozdag, R. Brewer, and E. Ekici, "Data harvesting with mobile elements in wireless sensor networks," Computer Networks, vol. 50, pp. 3449-3465, 2006.

[3] U. Lee, E. Magistretti, B. Zhou, M. Gerla, P. Bellavista, and A. Corradi, "Efficient data harvesting in mobile sensor platforms," in Fourth IEEE International Conference on Pervasive Computing and Communications Workshops (PERCOMW'06), 2006.

[4] E. Frazzoli and F. Bullo, "Decentralized algorithms for vehicle routing in a stochastic time-varying environment," in 43rd IEEE Conference on Decision and Control, December 2004, pp. 3357-3363.

[5] A. A. Somasundara, A. Ramamoorthy, and M. B. Srivastava, "Mobile element scheduling for efficient data collection in wireless sensor networks with dynamic deadlines," in Proceedings of the 25th IEEE International Real-Time Systems Symposium (RTSS'04), 2004.

[6] H. Takagi, Analysis of Polling Systems. The MIT Press, 1986.

[7] A. T. Klesh, A. R. Girard, and P. T. Kabamba, "Path planning for cooperative time-optimal information collection," in Proceedings of the American Control Conference, Seattle, June 2008.

[8] S. Meyn, Control Techniques for Complex Networks. Cambridge University Press, 2008.

[9] W.-M. Lan and T. L. Olsen, "Multiproduct systems with both setup times and costs: Fluid bounds and schedules," Operations Research, vol. 54, no. 3, pp. 505-522, May-June 2006.

[10] T. Cover and J. Thomas, Elements of information theory. Wiley New York, 1991

[11] J. Le Ny, "Performance optimization for unmanned vehicle systems," Ph.D. dissertation, Massachusetts Institute of Technology, 2008.

[12] A. Bryson and Y.-C. Ho, Applied Optimal Control: Optimization, Estimation, and Control. Taylor and Francis, 1975.

[13] P. Liu, R. Berry, and M. Honig, "A fluid analysis of a utilitybased wireless scheduling policy," IEEE Transactions on Information Theory, vol. 52, no. 7, pp. 2872-2889, 2006. 